\title{
New Photometric Observations of RS Oph
}

\author{
Irina Voloshina and Vladimir Metlov
}

Sternberg Astronomical Institute, Moscow State University,

Universitetskij prospect 13, Moscow 119992, Russia

email: vib@sai.msu.ru

\begin{abstract}
The recurrent nova RS Oph is a long period ( 455 days) binary system consisting of a hot white dwarf with mass close to the Chandrasekhar limit and an M-type giant secondary. Here we present the results of photometric observations of this nova which were made during recent years with the telescopes of the Sternberg Astronomical Institute in Crimea.
\end{abstract}

Keywords. stars: novae, cataclysmic variables, oscillations, individual (RS Oph)

The cataclysmic variable RS Oph together with three other systems (T CrB, V3800 Sgr, and V745 Sco) belong to the long period binaries subtype of recurrent novae, which resemble symbiotic stars in many respects. The primary component in these systems is a hot white dwarf, and the secondary is an M-type giant. RS Oph is a fast nova. This nova was discovered in 1898, and five other nova-like outbursts were detected during the history of this star with recurrence time $\sim 20$ years. During the outbursts the brightness of RS Oph typically changes from $12^{m} .5$ to $4^{m} .5$. It is a wide binary system ( $\mathrm{a}=$ $316.5 R_{\odot}$ ) with inclination about $33^{\circ}$ (for details see review by Anupama 2008). Rapid light variations with amplitude about $0^{m} .3$ were found by Dobrzycka et al. (1996).

The CCD observations of RS Oph were made with the goal of studying short time variability on the scale of minutes to hours during two observational seasons with the telescopes of Sternberg Astronomical Institute Crimean Station:
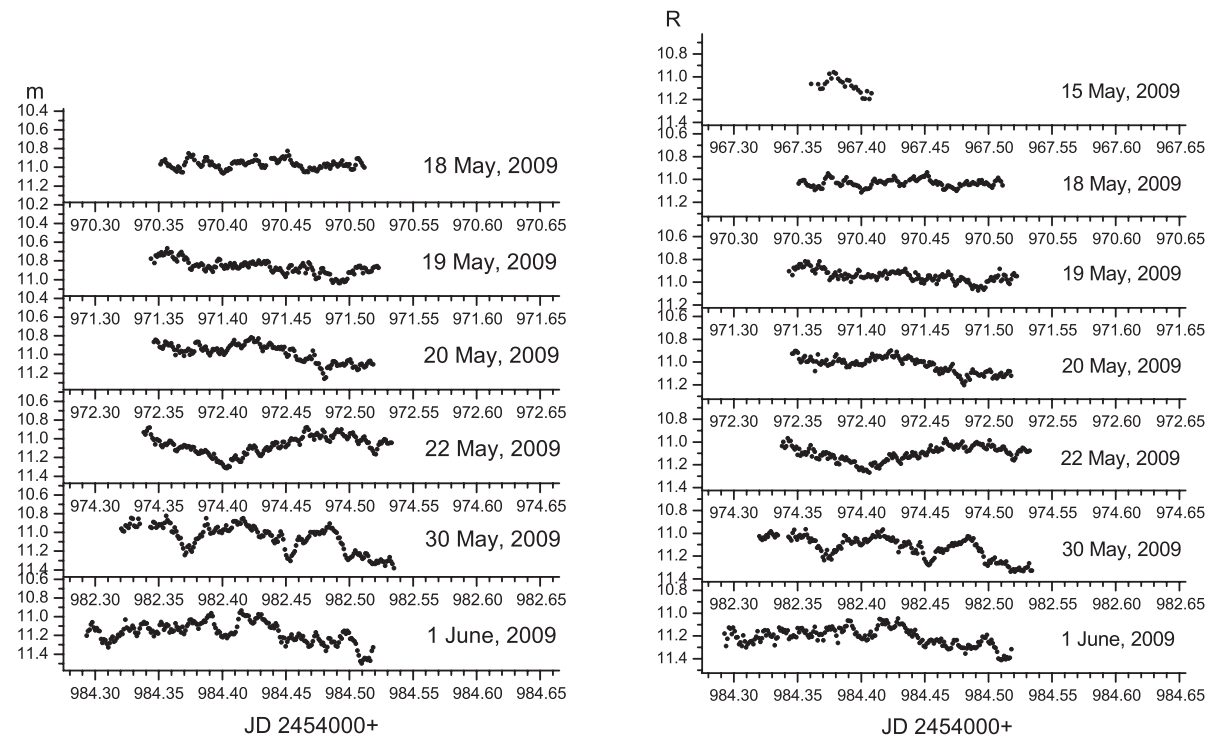

Figure 1. Individual light curves of RS Oph obtained in 2009: $V$ band (left) and $R$ (right). 

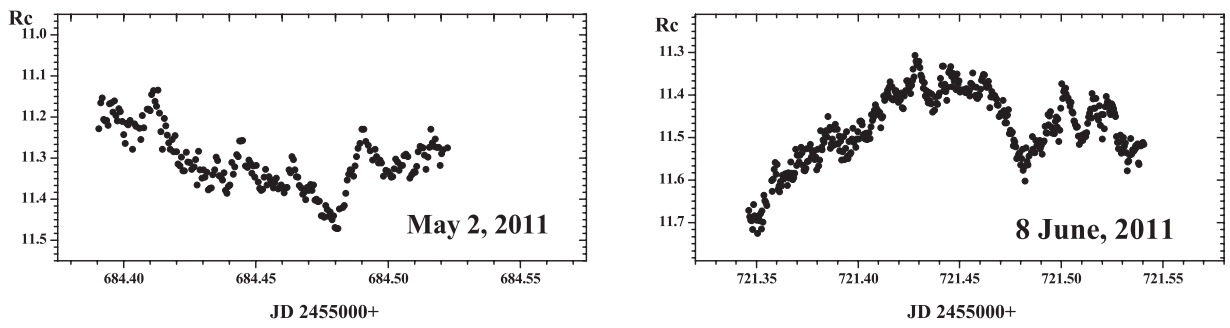

Figure 2. Some of the daily light curves of RS Oph obtained in 2011.
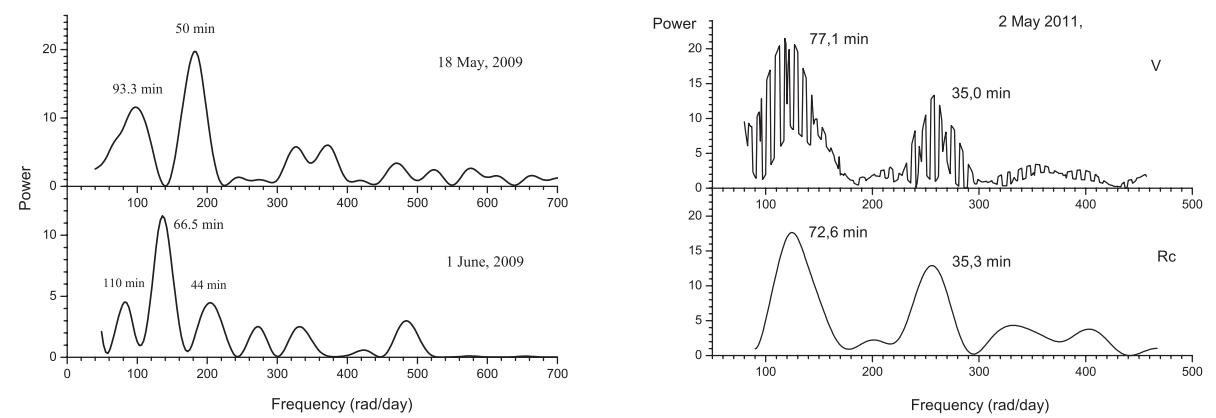

Figure 3. Lomb-Scargle periodogram of data showing periods obtained in 2009 and 2011.

2009: with 50-cm telescope + Pictor 416 CCD detector, $(512 \times 712$ pixels, 1 pixel $=$ $9 \mathrm{mkm})$ in $V$ band and 60-cm telescope + Ap 47 CCD detector, $(1024 \times 1024$ pixels, 1 pixel $=13 \mathrm{mkm}$ ) in $R$ band.

2011: with 60-cm telescope + Ap 47 CCD detector, $R_{c}$ band. The duration of an observational set is $\sim 4-5 \mathrm{hr}$. The star SAO 141899 was used as a local standard. More than 3000 frames were obtained in $V$ band and somewhat fewer in $R$ band for the period of observations. Aperture photometry was performed with MAXIM DL package.

A Lomb-Scargle period analysis of all the data shows the following rapid semi-coherent brightness variations in the light curves of RS Oph: variations with periods within 90-110 min range dominate in 2011, slightly changing from night to night. The peak corresponding to the 82 min period (Dobrzycka et al. 1996) was also present in the periodogram but with a smaller amplitude. Other groups of variations with periods 50-70 min and $\sim 35$ min dominate in 2009 light curves of RS Oph as in our previous study of RS Oph (Voloshina \& Metlov 2009).

Acknowledgements. This work is supported by Russian Fund of Basic Research under grant 09-02-00225a and State Support Program of Leading Scientific Schools of Russia (grant 7179.2010).

\section{References}

Anupama, G. C. 2008, in: A. Evans, M. F. Bode, T. J. O'Brien, \& M. J. Darnley (eds.), RS Ophiuchi (2006) and the Recurrent Nova Phenomenon, Proc. of the conference held 12-14 June, 2007, at Keele University, Keele, UK (San Francisco: ASP), 401 p. 31

Dobrzycka, D., Kenyon, S. J., \& Milone, A. A. E. 1996, AJ, 111, 414

Voloshina, I. \& Metlov V. 2009, in: B. Soonthornthum, S. Komonjinda, K. S.Cheng, \& K. C. Leung (eds.), The Eight Pacific Rim Conference on Stellar Astrophisics, Proc. of the conference held 5-9 May, 2008, Phuket, Thailand (San Francisco: ASP), 404 p. 72 